\title{
The Use of a Pruned Modular Decomposition for Maximum Matching Algorithms on Some Graph Classes
}

\section{Guillaume Ducoffe}

ICI - National Institute for Research and Development in Informatics, Bucharest, Romania The Research Institute of the University of Bucharest ICUB, Bucharest, Romania guillaume.ducoffe@ici.ro

\author{
Alexandru Popa \\ University of Bucharest, Bucharest, Romania \\ ICI - National Institute for Research and Development in Informatics, Bucharest, Romania \\ alexandru.popa@fmi.unibuc.ro
}

\begin{abstract}
We address the following general question: given a graph class $\mathcal{C}$ on which we can solve MAXIMUM MATChing in (quasi) linear time, does the same hold true for the class of graphs that can be modularly decomposed into $\mathcal{C}$ ? As a way to answer this question for distance-hereditary graphs and some other superclasses of cographs, we study the combined effect of modular decomposition with a pruning process over the quotient subgraphs. We remove sequentially from all such subgraphs their so-called one-vertex extensions (i.e., pendant, anti-pendant, twin, universal and isolated vertices). Doing so, we obtain a "pruned modular decomposition", that can be computed in quasi linear time. Our main result is that if all the pruned quotient subgraphs have bounded order then a maximum matching can be computed in linear time. The latter result strictly extends a recent framework in (Coudert et al., SODA'18). Our work is the first to explain why the existence of some nice ordering over the modules of a graph, instead of just over its vertices, can help to speed up the computation of maximum matchings on some graph classes.
\end{abstract}

2012 ACM Subject Classification Mathematics of computing $\rightarrow$ Graph theory, Theory of computation $\rightarrow$ Design and analysis of algorithms

Keywords and phrases maximum matching, FPT in P, modular decomposition, pruned graphs, one-vertex extensions, $P_{4}$-structure

Digital Object Identifier 10.4230/LIPIcs.ISAAC.2018.6

Related Version A full version of the paper is available at [14], https://arxiv.org/abs/1804. 09407.

Funding This work was supported by the Institutional research programme PN 1819 "Advanced IT resources to support digital transformation processes in the economy and society - RESINFOTD" (2018), project PN 1819-01-01 "Modeling, simulation, optimization of complex systems and decision support in new areas of IT\&C research", funded by the Ministry of Research and Innovation, Romania.

\section{Introduction}

Can we compute a maximum matching in a graph in linear-time? - i.e., computing a maximum set of pairwise disjoint edges in a graph. - Despite considerable years of research and the design of elegant combinatorial and linear programming techniques, the best-known

(c) (i) $\odot$ Guillaume Ducoffe and Alexandru Popa;

licensed under Creative Commons License CC-BY

29th International Symposium on Algorithms and Computation (ISAAC 2018).

Editors: Wen-Lian Hsu, Der-Tsai Lee, and Chung-Shou Liao; Article No. 6; pp. 6:1-6:13

Leibniz International Proceedings in Informatics

LI I ICS Schloss Dagstuhl - Leibniz-Zentrum für Informatik, Dagstuhl Publishing, Germany 
algorithms for this fundamental problem have stayed blocked to an $\mathcal{O}(m \sqrt{n})$-time complexity on $n$-vertex $m$-edge graphs [22]. Nevertheless, we can use some well-structured graph classes in order to overcome this superlinear barrier for particular cases of graphs. Our work combines two successful approaches for this problem, namely, the use of a vertex-ordering characterization for certain graph classes [5, 10, 21], and a recent technique based on the decomposition of a graph by its modules [9]. We detail these two approaches in what follows, before summarizing our contributions.

\section{$1.1 \quad$ Related work}

A cornerstone of most MAXimum MATCHING algorithms is the notion of augmenting paths [2, 15]. However, although we can compute a set of augmenting paths in linear-time [16], this is a tedious task that involves the technical notion of blossoms and this may need to be repeated $\Omega(\sqrt{n})$ times before a maximum matching can be computed [19]. A well-known greedy approach consists in, given some total ordering $\left(v_{1}, v_{2}, \ldots, v_{n}\right)$ over the vertices in the graph, to consider the exposed vertices $v_{i}$ by increasing order, then to try to match them with some exposed neighbour $v_{j}$ that appears later in the ordering [12]. The vertex $v_{j}$ can be chosen either arbitrarily or according to some specific rules depending on the graph class we consider. Our initial goal was to extend similar reduction rules to module-orderings.

Modular decomposition. A module in a graph $G=(V, E)$ is any vertex-subset $X$ such that every vertex of $V \backslash X$ is either adjacent to every of $X$ or nonadjacent to every of $X$. The modular decomposition of $G$ is a recursive decomposition of $G$ according to its modules [18]. We postpone its formal definition until Section 2. For now, we only want to stress that the vertices in the "quotient subgraphs" that are outputted by this decomposition represent modules of $G$ (e.g., see Fig. 1 for an insightful illustration). Our main motivation for considering modular decomposition in this note is its recent use in the field of parameterized complexity for polynomial problems. More precisely, let us call modular-width of a graph $G$ the minimum $k \geq 2$ such that every quotient subgraph in the modular decomposition of $G$ is either "degenerate" (i.e., complete or edgeless) or of order at most $k$. With Coudert, we proved in [9] that many "hard" graph problems in $\mathrm{P}$ - for which no linear-time algorithm is likely to exist - can be solved in $k^{\mathcal{O}(1)}(n+m)$-time on graphs with modular-width at most $k$. In particular, we proposed an $\mathcal{O}\left(k^{4} n+m\right)$-time algorithm for Maximum Matching.

One appealing aspect of our approach in [9] was that, for most problems studied, we obtained a linear-time reduction from the input graph $G$ to some (smaller) quotient subgraph $G^{\prime}$ in its modular decomposition. - We say that the problem is preserved by quotient. This paved the way to the design of efficient algorithms for these problems on graph classes with unbounded modular-width, assuming their quotient subgraphs are simple enough w.r.t. the problem at hands. We illustrated this possibility through the case of $(q, q-3)$-graphs (i.e., graphs where no set of at most $q$ vertices, $q \geq 7$, can induce more than $q-3$ paths of length four). However, this approach completely fell down for Maximum Matching. Indeed, our Maximum Matching algorithm in [9] works on supergraphs of the quotient graphs that need to be repeatedly updated every time a new augmenting path is computed. Such approach did not help much in exploiting the structure of quotient graphs. We managed to do so for $(q, q-3)$-graphs only through the help of a deeper structural theorem on the nontrivial modules in this class of graphs. Nevertheless, to take a shameful example, it was not even known before this work whether Maximum MATching could be solved faster than with the state-of-the art algorithms on graphs that can be modularly decomposed into paths! 


\subsection{Our contributions}

We propose pruning rules on the modules in a graph (some of them new and some others revisited) that can be used in order to compute Maximum MATching in linear-time on several new graph classes. More precisely, given a module $M$ in a graph $G=(V, E)$, recall that $M$ is corresponding to some vertex $v_{M}$ in a quotient graph $G^{\prime}$ of the modular decomposition of $G$. Assuming $v_{M}$ is a so-called one-vertex extension in $G^{\prime}$ (i.e., it is pendant, anti-pendant, universal, isolated or it has a twin), we show that a maximum matching for $G$ can be computed from a maximum matching of $G[M]$ and a maximum matching of $G \backslash M$ efficiently (see Section 4). Our rules are purely structural, in the sense that they only rely on the structural properties of $v_{M}$ in $G^{\prime}$ and not on any additional assumption on the nontrivial modules. Some of these rules (e.g., for isolated or universal modules) were first introduced in [9] - although with slightly different correctness proofs. Our main technical contributions in this work are the pruning rules for, respectively, pendant and anti-pendant modules (see Sections 4.2 and 4.3). The latter two cases are surprisingly the most intricate. In particular, they require amongst other techniques: the computation of specified augmenting paths of length up to 7 , the addition of some "virtual edges" in other modules, and a careful swapping between some matched and unmatched edges.

Then, we are left with pruning every quotient subgraph in the modular decomposition by sequentially removing the one-vertex extensions. We prove that the resulting "pruned quotient subgraphs" are unique (independent from the removal orderings) and that they can be computed in quasi linear-time using a trie data-structure (Section 3). Furthermore, as a case-study we prove that several superclasses of cographs are totally decomposable w.r.t. this new "pruned modular decomposition". These classes are further discussed in Section 5. Note that for some of them, such as distance-hereditary graphs, we so obtain the first known linear-time algorithm for MAXIMUM MATCHING - thereby extending previous partial results obtained for bipartite and chordal distance-hereditary graphs [10]. Our approach actually has similarities with a general greedy scheme applied to distance-hereditary graphs [7]. With slightly more work, we can extend our approach to every graph that can be modularly decomposed into cycles. The case of graphs of bounded modular treewidth [23] is left as an interesting open question.

Definitions and our first results are presented in Section 2. We introduce the pruned modular decomposition in Section 3, where we show that it can be computed in quasi linear-time. Then, the core of the paper is Section 4 where the pruning rules are presented along with their correctness proofs. In particular, we state our main result in Section 4.4. Applications of our approach to some graph classes are discussed in Section 5. Finally, we conclude in Section 6 with some open questions. Due to lack of space, several proofs are omitted. Full proofs can be found in our technical report [14].

\section{Preliminaries}

For the standard graph terminology, see [3]. We only consider graphs that are finite, simple and unweighted. For any graph $G=(V, E)$ let $n=|V|$ and $m=|E|$. Given a vertex $v \in V$, we denote its (open) neighbourhood by $N_{G}(v)=\{u \in V \mid\{u, v\} \in E\}$ and its closed neighbourhood by $N_{G}[v]=N_{G}(v) \cup\{v\}$. Similarly, we define the neighbourhood of any vertex-subset $S \subseteq V$ as $N_{G}(S)=\left(\bigcup_{v \in S} N_{G}(v)\right) \backslash S$. In what follows, we introduce our main algorithmic tool for the paper as well as the graph problems we study. 

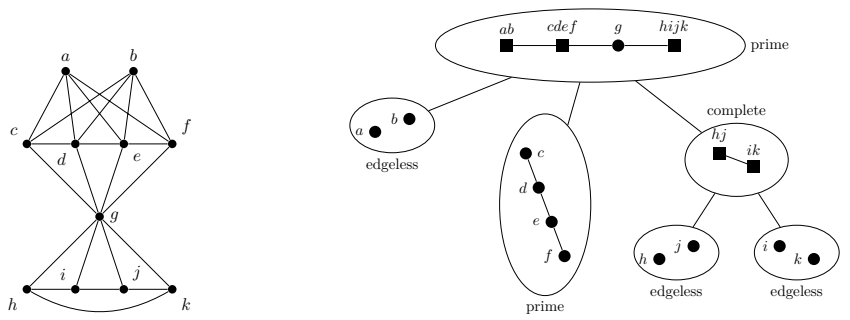

Figure 1 A graph and its modular decomposition.

\section{Modular decomposition}

A module in a graph $G=(V, E)$ is any subset $M \subseteq V(G)$ such that for any $u, v \in M$ we have $N_{G}(v) \backslash M=N_{G}(u) \backslash M$. There are trivial examples of modules such as $\emptyset, V$, and $\{v\}$ for every $v \in V$. Let $\mathcal{P}=\left\{M_{1}, M_{2}, \ldots, M_{p}\right\}$ be a partition of the vertex-set $V$. If for every $1 \leq i \leq p, M_{i}$ is a module of $G$, then we call $\mathcal{P}$ a modular partition of $G$. By abuse of notation, we will sometimes identify a module $M_{i}$ with the induced subgraph $H_{i}=G\left[M_{i}\right]$, i.e., we will write $\mathcal{P}=\left\{H_{1}, H_{2}, \ldots H_{p}\right\}$. The quotient subgraph $G / \mathcal{P}$ has vertex-set $\mathcal{P}$, and there is an edge between every two modules $M_{i}, M_{j} \in \mathcal{P}$ such that $M_{i} \times M_{j} \subseteq E$. Conversely, let $G^{\prime}=\left(V^{\prime}, E^{\prime}\right)$ be a graph and let $\mathcal{P}=\left\{H_{1}, H_{2}, \ldots H_{p}\right\}$. be a collection of subgraphs. The substitution graph $G^{\prime}(\mathcal{P})$ is obtained from $G^{\prime}$ by replacing every vertex $v_{i} \in V^{\prime}$ with a module inducing $H_{i}$. In particular, for $G^{\prime}={ }^{\operatorname{def}} G / \mathcal{P}$ we have that $G^{\prime}(\mathcal{P})=G$.

We say that $G$ is prime if its only modules are trivial (i.e., $\emptyset, V$, and the singletons $\{v\}$ ). We call a module $M$ strong if it does not overlap any other module, i.e., for any module $M^{\prime}$ of $G$, either one of $M$ or $M^{\prime}$ is contained in the other or $M$ and $M^{\prime}$ do not intersect. Let $\mathcal{M}(G)$ be the family of all inclusion wise maximal strong modules of $G$ that are proper subsets of $V$. The family $\mathcal{M}(G)$ is a modular partition of $G$ [18], and so, we can define $G^{\prime}=G / \mathcal{M}(G)$. The following structure theorem is due to Gallai.

- Theorem 1 ([17]). For an arbitrary graph $G$ exactly one of the following conditions is satisfied.

1. $G$ is disconnected;

2. its complement $\bar{G}$ is disconnected;

3. or its quotient graph $G^{\prime}=G / \mathcal{M}(G)$ is prime for modular decomposition.

We now formally define the modular decomposition of $G$-introduced earlier in Section 1. We output the quotient graph $G^{\prime}=G / \mathcal{M}(G)$ and, for any strong module $M \in \mathcal{M}(G)$ that is nontrivial (possibly none if $G=G^{\prime}$ ), we also output the modular decomposition of $G[M]$. By Theorem 1 the subgraphs from the modular decomposition are either edgeless, complete, or prime for modular decomposition. See Fig. 1 for an example. The modular decomposition of a given graph $G=(V, E)$ can be computed in linear-time [25]. There are many graph classes that can be characterized using the modular decomposition.In particular, $G$ is a cograph if and only if every quotient subgraph in its modular decomposition is either complete or disconnected [8].

\section{Maximum Matching}

A matching in a graph is defined as a set of edges with pairwise disjoint end vertices. The maximum cardinality of a matching in a given graph $G=(V, E)$ is denoted by $\mu(G)$. 
- Problem 2 (Maximum Matching).

Input: $A$ graph $G=(V, E)$.

Output: $A$ matching of $G$ with maximum cardinality.

We remind the reader that Maximum Matching can be solved in $\mathcal{O}(m \sqrt{n})$-time on general graphs [22] - although we do not use this result directly in our paper. Furthermore, let $G=(V, E)$ be a graph and let $F \subseteq E$ be a matching of $G$. We call a vertex matched if it is incident to an edge of $F$, and exposed otherwise. Then, we define an $F$-augmenting path as a path where the two ends are exposed, and the edges belong alternatively to $F$ and not to $F$. It is well-known and easy to check that, given an $F$-augmenting path $P$, the matching $E(P) \Delta F$ (obtained by symmetric difference on the edges) has larger cardinality than $F$.

- Lemma 3 (Berge, [2]). A matching $F$ in $G=(V, E)$ is maximum if and only if there is no F-augmenting path.

In this paper, we will consider an intermediate matching problem, first introduced in [9].

- Problem 4 (Module Matching).

Input: A graph $G^{\prime}=\left(V^{\prime}, E^{\prime}\right)$ with the following additional information;

- a collection of subgraphs $\mathcal{P}=\left\{H_{1}, H_{2}, \ldots, H_{p}\right\}$

- a collection $\mathcal{F}=\left\{F_{1}, F_{2}, \ldots, F_{p}\right\}$, with $F_{i}$ being a maximum matching of $H_{i}$ for every $i$.

Output: $A$ matching of $G=G^{\prime}(\mathcal{P})$ with maximum cardinality.

A natural choice for Module Matching would be to take $\mathcal{P}=\mathcal{M}(G)$. However, we will allow $\mathcal{P}$ to take different values for our reduction rules.

Additional notations. Let $\left\langle G^{\prime}, \mathcal{P}, \mathcal{F}\right\rangle$ be any instance of Module Matching. The order of $G^{\prime}$, equivalently the cardinality of $\mathcal{P}$, is denoted by $p$. For every $1 \leq i \leq p$ let $M_{i}=V\left(H_{i}\right)$ and let $n_{i}=\left|M_{i}\right|$ be the order of $H_{i}$. We denote $\delta_{i}=\left|E\left(M_{i}, \overline{M_{i}}\right)\right|$ the size of the cut $E\left(M_{i}, \overline{M_{i}}\right)$ with all the edges between $M_{i}$ and $N_{G}\left(M_{i}\right)$. In particular, we have $\delta_{i}=\sum_{v_{j} \in N_{G^{\prime}}\left(v_{i}\right)} n_{i} n_{j}$. Let us define $\Delta m\left(G^{\prime}\right)=\sum_{i=1}^{p} \delta_{i}$. We will omit the dependency in $G^{\prime}$ if it is clear from the context. Finally, let $\Delta \mu=\mu(G)-\sum_{i=1}^{p} \mu\left(H_{i}\right)$.

Our framework is based on the following lemma (inspired from [9]).

- Lemma 5. Let $G=(V, E)$ be a graph. Suppose that for every $H^{\prime}$ in the modular decomposition of $G$ we can solve Module MATCHIng on any instance $\left\langle H^{\prime}, \mathcal{P}, \mathcal{F}\right\rangle$ in time $T(p, \Delta m, \Delta \mu)$, where $T$ is a subadditive function ${ }^{1}$. Then, we can solve MAXIMUM MATCHING on $G$ in time $\mathcal{O}(T(\mathcal{O}(n), m, n))$.

An important observation for our subsequent analysis is that, given any module $M$ of a graph $G$, the internal structure of $G[M]$ has no more relevance after we computed a maximum matching $F_{M}$ for this subgraph. More precisely, we will use the following lemma:

- Lemma 6 ([9]). Let $M$ be a module of $G=(V, E)$, let $G[M]=\left(M, E_{M}\right)$ and let $F_{M} \subseteq E_{M}$ be a maximum matching of $G[M]$. Then, every maximum matching of $G_{M}^{\prime}=\left(V,\left(E \backslash E_{M}\right) \cup\right.$ $\left.F_{M}\right)$ is a maximum matching of $G$.

By Lemma 6 we can modify our algorithmic framework as follows. For every instance $\left\langle G^{\prime}, \mathcal{P}, \mathcal{F}\right\rangle$ for Module Matching, we can assume that $H_{i}=\left(M_{i}, F_{i}\right)$ for every $1 \leq i \leq p$.

1 We stress that every polynomial function is subadditive. 
Data structures. Finally, let $\left\langle G^{\prime}, \mathcal{P}, \mathcal{F}\right\rangle$ be any instance for Module Matching. A $c a$ nonical ordering of $H_{i}$ (w.r.t. $F_{i}$ ) is a total ordering over $V\left(H_{i}\right)$ such that the exposed vertices appear first, and every two vertices that are matched together are consecutive. In what follows, we will assume that we have access to a canonical ordering for every $i$. Such orderings can be computed in time $\mathcal{O}\left(\sum_{i}\left|M_{i}\right|+\left|F_{i}\right|\right)$ by scanning all the modules and the matchings in $\mathcal{F}$, that is an $\mathcal{O}(\Delta m)$ provided $G^{\prime}$ has no isolated vertex.

Furthermore, let $F$ be a (not necessarily maximum) matching for the subdivision $G=$ $G^{\prime}(\mathcal{P})$. We will make the standard assumption that, for every $v \in V(G)$, we can decide in constant-time whether $v$ is matched by $F$, and if so, we can also access in constant-time to the vertex matched with $v$.

\section{A pruned modular decomposition}

In this section, we introduce a pruning process over the quotient subgraphs, that we use in order to refine the modular decomposition.

Definition 7. Let $G=(V, E)$ be a graph. We call $v \in V$ a one-vertex extension if it falls in one of the following cases:

- $N_{G}[v]=V$ (universal $)$ or $N_{G}(v)=\emptyset($ isolated $)$;

- $N_{G}[v]=V \backslash u$ (anti-pendant) or $N_{G}(v)=\{u\}$ (pendant), for some $u \in V \backslash v$;

- $N_{G}[v]=N_{G}[u]$ (true twin) or $N_{G}(v)=N_{G}(u)$ (false twin), for some $u \in V \backslash v$.

A pruned subgraph of $G$ is obtained from $G$ by sequentially removing one-vertex extensions (in the current subgraph) until it can no more be done. This terminology was introduced in [20], where they only considered the removals of twin and pendant vertices. Also, the clique-width of graphs that are totally decomposed by the above pruning process (i.e., with their pruned subgraph being a singleton) was studied in $[24]^{2}$. Our contribution in this part is twofold. First, we show that the gotten subgraph is "almost" independent of the removal ordering, i.e., there is a unique pruned subgraph of $G$ (up to isomorphism). The latter can be derived from the following (easy) lemma:

- Lemma 8. Let $G=(V, E)$ be a graph and let $v, v^{\prime} \in V$ be one-vertex extensions of $G$. If $v, v^{\prime}$ are not pairwise twins then $v^{\prime}$ is a one-vertex extension of $G \backslash v$.

- Corollary 9. Every graph $G=(V, E)$ has a unique pruned subgraph up to isomorphism.

For many graph classes a pruning sequence can be computed in linear-time. We observe that the same can be done for any graph (up to a logarithmic factor).

- Proposition 10. For every graph $G=(V, E)$, we can compute a pruned subgraph in $\mathcal{O}(n+m \log n)$-time.

Proof. By Corollary 9, we are left with greedily searching for, then eliminating, the one-vertex extensions. We can compute the ordered degree sequence of $G$ in $\mathcal{O}(n+m)$-time. Furthermore, after any vertex $v$ is eliminated, we can update this sequence in $\mathcal{O}(|N(v)|)$-time. Hence, up to a total update time in $\mathcal{O}(n+m)$, at any step we can detect and remove in constant-time any vertex that is either universal, isolated, pendant or anti-pendant. Finally, in [20] they proposed a trie data-structure supporting the following two operations: suppression of a vertex; and detection of true or false twins (if any). The total time for all the operations on this data-structure is in $\mathcal{O}(n+m \log n)[20]$.

2 Anti-twins are also defined as one-vertex extensions in [24]. Their integration to this framework remains to be done. 
We will term "pruned modular decomposition" of a graph $G$ the collection of the pruned subgraphs for all the quotient subgraphs in the modular decomposition of $G$. Note that there is a unique pruned modular decomposition of $G$ (up to isomorphism) and that it can be computed in $\mathcal{O}(n+m \log n)$-time by Proposition 10 (applied to every quotient subgraph in the modular decomposition separately). Furthermore, we remark that most cases of one-vertex extensions imply the existence of non trivial modules, and so, they cannot exist in the prime quotient subgraphs of the modular decomposition. Nevertheless, such vertices may appear after removal of pendant or anti-pendant vertices (e.g., in the bull graph).

\section{Reduction rules}

Let $\left\langle G^{\prime}, \mathcal{P}, \mathcal{F}\right\rangle$ be any instance of Module Matching. Suppose that $v_{1}$, the vertex corresponding to $M_{1}$ in $G^{\prime}$, is a one-vertex extension. Under this assumption, we present reduction rules to a smaller instance $\left\langle G^{*}, \mathcal{P}^{*}, \mathcal{F}^{*}\right\rangle$ where $\left|\mathcal{P}^{*}\right|<|\mathcal{P}|$. Each rule can be implemented to run in $\mathcal{O}\left(\Delta m\left(G^{\prime}\right)-\Delta m\left(G^{*}\right)\right)$-time. Due to lack of space, we skip the complexity analysis.

In Section 4.1 we recall the rules introduced in [9] for universal and isolated modules (explicitly) and for false or true twin modules (implicitly). Our main technical contributions are the reduction rules for pendant and anti-pendant modules (in Sections 4.2 and 4.3, respectively), which are surprisingly the most intricate. Finally, we end this section stating our main result (Theorem 29).

\subsection{Simple cases}

We introduce two local operations on a matching, first used in [26] for cographs. Let $F \subseteq E$ be a matching and let $M \subseteq V$ be a module.

- Operation 11 (MATCH). While there are $x \in M, y \in N(M)$ exposed, add $\{x, y\}$ to $F$.

- Operation 12 (SPLIT). While there are $x, x^{\prime} \in M, y, y^{\prime} \in N(M)$ such that $x$ and $x^{\prime}$ are exposed, and $\left\{y, y^{\prime}\right\} \in F$, replace $\left\{y, y^{\prime}\right\}$ in $F$ by $\{x, y\},\left\{x^{\prime}, y^{\prime}\right\}$.

Let $G=H_{1} \oplus H_{2}$ be the join of the two graphs $H_{1}, H_{2}$ and let $F_{1}, F_{2}$ be maximum matchings for $H_{1}, H_{2}$, respectively. The "MATCH and SPLIT" technique consists in applying Operations 11 then 12 to $M=V\left(H_{1}\right)$ and $F=F_{1} \cup F_{2}$, thereby obtaining a new matching $F^{\prime}$, then to $M=V\left(H_{2}\right)$ and $F=F^{\prime}$. Based on this technique, we design the following rules:

- Reduction rule 13 (see also [9]). Suppose $v_{1}$ is isolated in $G^{\prime}$. We set $G^{*}=G^{\prime} \backslash v_{1}$, $\mathcal{P}^{*}=\mathcal{P} \backslash\left\{H_{1}\right\}$, and $\mathcal{F}^{*}=\mathcal{F} \backslash\left\{F_{1}\right\}$. Furthermore, let $F^{*}$ be a maximum matching of $G^{*}\left(\mathcal{P}^{*}\right)=G\left[V \backslash M_{1}\right]$. We output $F^{*} \cup F_{1}$.

- Reduction rule 14 (see also [9]). Suppose $v_{1}$ is universal in $G^{\prime}$. We set $G^{*}=G \backslash v_{1}$, $\mathcal{P}^{*}=\mathcal{P} \backslash\left\{H_{1}\right\}, \mathcal{F}^{*}=\mathcal{F} \backslash\left\{F_{1}\right\}$. Furthermore, let $F^{*}$ be a maximum matching of the subdivision $G^{*}\left(\mathcal{P}^{*}\right)=G\left[V \backslash M_{1}\right]$. We apply the "MATCH and SPLIT" technique to $M_{1}, F_{1}$ with $V \backslash M_{1}, F^{*}$.

- Reduction rule 15. Suppose $v_{1}, v_{2}$ are false twins in $G^{\prime}$. We set $G^{*}=G^{\prime} \backslash v_{1}, \mathcal{P}^{*}=$ $\left\{H_{1} \cup H_{2}\right\} \cup\left(\mathcal{P} \backslash\left\{H_{1}, H_{2}\right\}\right), \mathcal{F}^{*}=\left\{F_{1} \cup F_{2}\right\} \cup\left(\mathcal{F} \backslash\left\{F_{1}, F_{2}\right\}\right)$. We output a maximum matching of $G^{*}\left(\mathcal{P}^{*}\right)=G$.

- Reduction rule 16. Suppose $v_{1}, v_{2}$ are true twins in $G^{\prime}$. Let $F_{2}^{*}$ be the matching of $H_{1} \oplus H_{2}$ obtained from the "MATCH and SPLIT" technique applied to $M_{1}, F_{1}$ with $M_{2}, F_{2}$. We set $G^{*}=G \backslash v_{1}, \mathcal{P}^{*}=\left\{H_{1} \oplus H_{2}\right\} \cup\left(\mathcal{P} \backslash\left\{H_{1}, H_{2}\right\}\right), \mathcal{F}^{*}=\left\{F_{2}^{*}\right\} \cup\left(\mathcal{F} \backslash\left\{F_{1}, F_{2}\right\}\right)$. We output a maximum matching of $G^{*}\left(\mathcal{P}^{*}\right)=G$. 


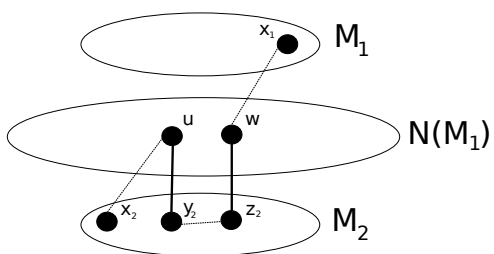

Figure 2 An augmenting path of length 5 with ends $x_{1}, x_{2}$. Matched edges are drawn in bold.

\subsection{Anti-pendant}

Suppose $v_{1}$ is anti-pendant in $G^{\prime}$. W.l.o.g., $v_{2}$ is the unique vertex that is nonadjacent to $v_{1}$ in $G^{\prime}$. By Lemma 6, we can also assume w.l.o.g. that $E\left(H_{i}\right)=F_{i}$ for every $i$. In this situation, we start applying Reduction rule 13, i.e., we set $G^{*}=G^{\prime} \backslash v_{1}, \mathcal{P}^{*}=\mathcal{P} \backslash\left\{H_{1}\right\}$, $\mathcal{F}^{*}=\mathcal{F} \backslash\left\{F_{1}\right\}$. Then, we obtain a maximum matching $F^{*}$ of $G \backslash M_{1}$ (i.e., by applying our reduction rules to this new instance). Finally, from $F_{1}$ and $F^{*}$, we compute a maximum matching $F$ of $G$, using an intricate procedure. We detail this procedure next.

First phase: pre-processing. Our correctness proofs in what follows will assume that some additional properties hold on the matched vertices in $F^{*}$. So, we start correcting the initial matching $F^{*}$ so that it is the case. For that, we introduce two "swapping" operations. Recall that $v_{2}$ is the unique vertex that is nonadjacent to $v_{1}$ in $G^{\prime}$.

- Operation 17 (REPAIR). While there exist $x_{2}, y_{2} \in M_{2}$ such that $\left\{x_{2}, y_{2}\right\} \in F_{2}$ and $y_{2}$ is exposed in $F^{*}$, we replace any edge $\left\{x_{2}, w\right\} \in F^{*}$ by $\left\{x_{2}, y_{2}\right\}$.

- Operation 18 (ATTRACT). While there exist $x_{2} \in M_{2}$ exposed and $\{u, w\} \in F^{*}$ such that $u \in N_{G}\left(M_{2}\right), w \notin M_{2}$, we replace $\{u, w\}$ by $\left\{u, x_{2}\right\}$.

Let $F^{(0)}=F_{1} \cup F^{*}$. Summarizing, we get:

- Definition 19. A matching $F$ of $G$ is good if it satisfies the following two properties:

1. every vertex matched by $F_{1} \cup F_{2}$ is also matched by $F$;

2. either every vertex in $M_{2}$ is matched, or there is no matched edge in $N_{G}\left(M_{2}\right) \times N_{G}\left(M_{1}\right)$.

- Fact 20. $F^{(0)}$ is a good matching of $G$.

Main phase: a modified Match and Split. We now apply the following three operations sequentially:

1. $\operatorname{Match}\left(M_{1}, F^{(0)}\right)$ (Operation 11). Doing so, we obtain a larger good matching $F^{(1)}$.

2. $\operatorname{SpLit}\left(M_{1}, F^{(1)}\right)$ (Operation 12). Doing so, we obtain a larger good matching $F^{(2)}$.

3. the operation UNBREAK, defined in what follows (see also Fig. 2 for an illustration):

- Operation 21 (UnBreAK). While there exist $x_{1} \in M_{1}$ and $x_{2} \in M_{1} \cup M_{2}$ exposed, and there also exist $\left\{y_{2}, z_{2}\right\} \in F_{2} \backslash F^{(2)}$, we replace any two edges $\left\{y_{2}, u\right\},\left\{z_{2}, w\right\} \in F^{(2)}$ by the three edges $\left\{x_{2}, u\right\},\left\{y_{2}, z_{2}\right\}$ and $\left\{w, x_{1}\right\}$.

We stress that the two edges $\left\{y_{2}, u\right\},\left\{z_{2}, w\right\} \in F^{(2)}$ always exist since $F^{(2)}$ is a good matching of $G$. Furthermore doing so, we obtain a larger matching $F^{(3)}$.

The resulting matching $F^{(3)}$ is not necessarily maximum. However, this matching satisfies the following crucial property:

- Lemma 22. No vertex of $M_{1}$ can be an end in an $F^{(3)}$-augmenting path. 


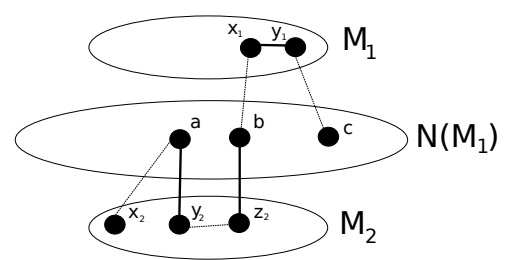

Figure 3 An augmenting path of length 7 with ends $x_{2}, c$. Matched edges are drawn in bold.

Finalization phase: breaking some edges in $\boldsymbol{F}_{\mathbf{1}}$. Intuitively, the matching $F^{(3)}$ may not be maximum because we sometimes need to borrow some edges of $F_{1}$ in augmenting paths. So, we complete our procedure by performing the following two operations: Let $U_{1}$ contain all the exposed vertices in $N\left(M_{1}\right)$. Consider the subgraph $G\left[M_{1} \cup U_{1}\right]=G\left[M_{1}\right] \oplus G\left[U_{1}\right]$. The set $U_{1}$ is a module of this subgraph. We apply $\operatorname{Split}\left(U_{1}, F^{(3)}\right)$ in $G\left[M_{1} \cup U_{1}\right]$. Doing so, we obtain a larger good matching $F^{(4)}$. Then, we apply LocALAUG, defined next (see also Fig. 3 for an illustration):

- Operation 23 (LocAlAug). While there exist $x_{2} \in M_{2}$ and $c \in N\left(M_{1}\right)$ exposed, and there also exist $\left\{x_{1}, y_{1}\right\} \in F_{1} \cap F^{(4)}$ and $\left\{y_{2}, z_{2}\right\} \in F_{2} \backslash F^{(4)}$, we do the following:

- we remove $\left\{x_{1}, y_{1}\right\}$ and any edge $\left\{a, y_{2}\right\},\left\{b, z_{2}\right\}$ from $F^{(4)}$;

- we add $\left\{x_{2}, a\right\},\left\{y_{2}, z_{2}\right\},\left\{b, x_{1}\right\}$ and $\left\{y_{1}, c\right\}$ in $F^{(4)}$.

We stress that the two edges $\left\{y_{2}, a\right\},\left\{z_{2}, b\right\} \in F^{(4)}$ always exist since $F^{(4)}$ is a good matching of $G$. Furthermore doing so, we obtain a larger matching $F^{(5)}$.

- Lemma 24. $F^{(5)}$ is a maximum-cardinality matching of $G$.

\subsection{Pendant}

Suppose $v_{1}$ is pendant in $G^{\prime}$. W.l.o.g., $v_{2}$ is the unique vertex that is adjacent to $v_{1}$ in $G^{\prime}$. This last case is arguably more complex than the others since it requires both a pre-processing and a post-processing treatment on the matching.

First phase: greedy matching. We apply the "MATCH and SPLIT" technique to $M_{1}$. Doing so, we obtain a set $F_{1,2}$ of matched edges between $M_{1}$ and $M_{2}$. We remove $V\left(F_{1,2}\right)$, the set of vertices incident to an edge of $F_{1,2}$, from $G$. Then, there are three cases. If $M_{2} \subseteq V\left(F_{1,2}\right)$ then $M_{1} \backslash V\left(F_{1,2}\right)$ is isolated. We apply Reduction rule 13. If $M_{1} \subseteq V\left(F_{1,2}\right)$ then $M_{1}$ is already eliminated. The interesting case is when both $M_{1} \backslash V\left(F_{1,2}\right)$ and $M_{2} \backslash V\left(F_{1,2}\right)$ are nonempty. In particular, suppose there remains an exposed vertex $x_{1} \in M_{1} \backslash V\left(F_{1,2}\right)$. Since $M_{2} \backslash V\left(F_{1,2}\right) \neq \emptyset$, there exists $\left\{x_{2}, y_{2}\right\} \in F_{2}$ such that $x_{2}, y_{2} \notin V\left(F_{1,2}\right)$. We remove $x_{1}$ from $M_{1}, x_{2}$ from $M_{2},\left\{x_{2}, y_{2}\right\}$ from $F_{2}$ and then we add $\left\{x_{1}, x_{2}\right\}$ in $F_{1,2}$. Our first result in this section is that there always exists an optimal solution that contains $F_{1,2}$. This justifies a posteriori the removal of $V\left(F_{1,2}\right)$ from $G$.

Lemma 25. There is a maximum matching of $G$ that contains all edges in $F_{1,2}$.

We stress that during this phase, all the operations except maybe the last one increase the cardinality of the matching. Furthermore, the only possible operation that does not increase the cardinality of the matching is the replacement of an edge in $F_{2}$ by an edge in $F_{1,2}$. Doing so, either we fall in one of the two pathological cases $M_{1} \subseteq V\left(F_{1,2}\right)$ or $M_{2} \subseteq V\left(F_{1,2}\right)$ (easy to solve), or then we obtain through the replacement operation the following stronger property:

- Property 26. All vertices in $M_{1}$ are matched by $F_{1}$. 
We will assume Property 26 to be true for the remaining of this section.

Second phase: virtual split edges. We complete the previous phase by performing a SPLIT between $M_{2}, M_{1}$ (Operation 12). That is, while there exist two exposed vertices $x_{2}, y_{2} \in M_{2}$ and a matched edge $\left\{x_{1}, y_{1}\right\} \in F_{1}$ we replace $\left\{x_{1}, y_{1}\right\}$ by $\left\{x_{1}, x_{2}\right\},\left\{y_{1}, y_{2}\right\}$ in the current matching. However, we encode the SpLiT operation using virtual edges in $H_{2}$. Formally, we add a virtual edge $\left\{x_{2}, y_{2}\right\}$ in $H_{2}$ that is labeled by the corresponding edge $\left\{x_{1}, y_{1}\right\} \in F_{1}$. Let $H_{2}^{*}$ and $F_{2}^{*}$ be obtained from $H_{2}$ and $F_{2}$ by adding all the virtual edges. We set $G^{*}=G^{\prime} \backslash v_{1}$, $\mathcal{P}^{*}=\left\{H_{2}^{*}\right\} \cup\left(\mathcal{P} \backslash\left\{H_{1}, H_{2}\right\}\right)$ and $\mathcal{F}^{*}=\left\{F_{2}^{*}\right\} \cup\left(\mathcal{F} \backslash\left\{F_{1}, F_{2}\right\}\right)$.

Intuitively, virtual edges are used in order to shorten the augmenting paths crossing $M_{1}$.

Third phase: post-processing. Let $F^{*}$ be a maximum-cardinality matching of the subdivision $G^{*}\left(\mathcal{P}^{*}\right)$ (i.e., obtained by applying our reduction rules to the new instance). We construct a matching $F$ for $G$ as follows. We add in $F$ all the non virtual edges in $F^{*}$. For every virtual edge $\left\{x_{2}, y_{2}\right\}$, let $\left\{x_{1}, y_{1}\right\} \in F_{1}$ be its label. If $\left\{x_{2}, y_{2}\right\} \in F^{*}$ then we add $\left\{x_{1}, y_{2}\right\},\left\{x_{2}, y_{1}\right\}$ in $F$, otherwise we add $\left\{x_{1}, y_{1}\right\}$ in $F$. In the first case, we say that we confirm the Split operation, whereas in the second case we say that we cancel it. Finally, we complete $F$ with all the edges of $F_{1}$ that do not label any virtual edge (i.e., unused during the second phase).

- Lemma 27. $F$ is a maximum-cardinality matching of $G$.

The above result is proved by contrapositive. More precisely, we prove intricate properties on the intersection of shortest augmenting paths with pendant modules. Using these properties and the virtual edges, we could transform any shortest $F$-augmenting path into an $F^{*}$-augmenting path, a contradiction.

\subsection{Main result}

Our framework consists in applying any reduction rule presented in this section until it can no more be done. Then, we rely on the following result:

- Theorem 28 ([9]). We can solve Module Matching for $\left\langle G^{\prime}, \mathcal{P}, \mathcal{F}\right\rangle$ in $\mathcal{O}\left(\Delta \mu \cdot p^{4}\right)$-time.

We are now ready to state our main result in this paper (the proof of which directly follows from all the previous results in this section).

- Theorem 29. Let $G=(V, E)$ be a graph. Suppose that, for every prime subgraph $H^{\prime}$ in the modular decomposition of $G$, its pruned subgraph has order at most $k$. Then, we can solve Maximum Matching for $G$ in $\mathcal{O}\left(k^{4} \cdot n+m \log n\right)$-time.

\section{Applications}

We conclude this paper presenting applications and refinements of our main result to some graph classes. Recall that cographs are exactly the graphs that are totally decomposable by modular decomposition [8]. We start showing that several distinct generalizations of cographs in the literature are totally decomposable by the pruned modular decomposition. 
Distance-hereditary graphs. A graph $G=(V, E)$ is distance-hereditary if it can be reduced to a singleton by pruning sequentially the pendant vertices and twin vertices [1]. Conversely, $G$ is co-distance hereditary if it is the complement of a distance-hereditary graph, i.e., it can be reduced to a singleton by pruning sequentially the anti-pendant vertices and twin vertices. In both cases, the corresponding pruning sequence can be computed in linear-time $[11,13]$. Therefore, we can derive the following result from our framework:

- Proposition 30. We can solve MAximum Matching in linear-time on graphs that can be modularly decomposed into distance-hereditary graphs and co-distance hereditary graphs.

Trees are a special subclass of distance-hereditary graphs. We say that a graph has modular treewidth at most $k$ if every prime quotient subgraph in its modular decomposition has treewidth at most $k$. In particular, graphs with modular treewidth at most one are exactly the graphs that can be modularly decomposed into trees ${ }^{3}$. We stress the following consequence of Proposition 30:

- Corollary 31. We can solve MAXIMUM MATCHING in linear-time on graphs with modulartreewidth at most one.

The case of graphs with modular treewidth $k \geq 2$ is left as an intriguing open question.

Tree-perfect graphs. Two graphs $G_{1}, G_{2}$ are $P_{4}$-isomorphic if there exists a bijection from $G_{1}$ to $G_{2}$ such that a 4 -tuple induces a $P_{4}$ in $G_{1}$ if and only if its image in $G_{2}$ also induces a $P_{4}$ [6]. The notion of $P_{4}$-isomorphism plays an important role in the study of perfect graphs. A graph is tree-perfect if it is $P_{4}$-isomorphic to a tree [4]. We prove the following result:

- Proposition 32. Tree-perfect graphs are totally decomposable by the pruned modular decomposition. In particular, we can solve MAXIMUM MATCHING in linear-time on treeperfect graphs.

Our proof is based on a deep structural characterization of tree-perfect graphs [4].

The case of unicycles. We end up this section with a refinement of our framework for the special case of unicyclic quotient graphs (a.k.a., graphs with exactly one cycle).

- Proposition 33. We can solve Maximum Matching in linear-time on the graphs that can be modularly decomposed into unicycles.

For that, we reduce the case of unicycles to the case of cycles (removing pendant modules). Then, we test for all possible numbers of matched edges between two adjacent modules. Doing so, we reduce the case of cycles to the case of paths.

\section{Open problems}

The pruned modular decomposition happens to be an interesting add up in the study of Maximum Matching algorithms. An exhaustive study of its other algorithmic applications remains to be done. Moreover, another interesting question is to characterize the graphs that are totally decomposable by this new decomposition. We note that our pruning process can

\footnotetext{
${ }^{3}$ Our definition is more restricted than the one in [23] since they only impose the quotient subgraph $G^{\prime}$ to have bounded treewidth.
} 
be seen as a repeated update of the modular decomposition of a graph after some specified modules (pendant, anti-pendant) are removed. However, we can only detect a restricted family of these new modules (i.e., universal, isolated, twins). A fully dynamic modular decomposition algorithm could be helpful in order to further refine our framework.

\section{References}

1 H.-J. Bandelt and H. Mulder. Distance-hereditary graphs. J. of Combinatorial Theory, Series B, 41(2):182-208, 1986.

2 C. Berge. Two theorems in graph theory. Proceedings of the National Academy of Sciences, 43(9):842-844, 1957.

3 J. A. Bondy and U. S. R. Murty. Graph theory. Grad. Texts in Math., 2008.

4 A. Brandstädt and V. Le. Tree-and forest-perfect graphs. Discrete applied mathematics, 95(1-3):141-162, 1999.

5 M. Chang. Algorithms for maximum matching and minimum fill-in on chordal bipartite graphs. In ISAAC, pages 146-155. Springer, 1996.

6 V. Chvátal. A semi-strong perfect graph conjecture. In North-Holland mathematics studies, volume 88, pages 279-280. Elsevier, 1984.

7 O. Cogis and E. Thierry. Computing maximum stable sets for distance-hereditary graphs. Discrete Optimization, 2(2):185-188, 2005.

8 D. Corneil, Y. Perl, and L. Stewart. A linear recognition algorithm for cographs. SIAM Journal on Computing, 14(4):926-934, 1985.

9 D. Coudert, G. Ducoffe, and A. Popa. Fully polynomial FPT algorithms for some classes of bounded clique-width graphs. In SODA'18, pages 2765-2784. SIAM, 2018.

10 E. Dahlhaus and M. Karpinski. Matching and multidimensional matching in chordal and strongly chordal graphs. Discrete Applied Mathematics, 84(1-3):79-91, 1998.

11 G. Damiand, M. Habib, and C. Paul. A simple paradigm for graph recognition: application to cographs and distance hereditary graphs. Theoretical Computer Science, 263(1-2):99-111, 2001.

12 F. Dragan. On greedy matching ordering and greedy matchable graphs. In $W G^{\prime} 9^{\prime} 7$, volume 1335 of LNCS, pages 184-198. Springer, 1997.

13 S. Dubois, V. Giakoumakis, and C. El Mounir. On co-distance hereditary graphs. In $C T W$, pages $94-97,2008$.

14 G. Ducoffe and A. Popa. The use of a pruned modular decomposition for Maximum Matching algorithms on some graph classes. Technical Report arXiv:1804.09407, arXiv, 2018 .

15 J. Edmonds. Paths, trees, and flowers. Canadian J. of mathematics, 17(3):449-467, 1965.

16 H. Gabow and R. Tarjan. A linear-time algorithm for a special case of disjoint set union. In STOC'83, pages 246-251. ACM, 1983.

17 Tibor Gallai. Transitiv orientierbare graphen. Acta Math. Hungarica, 18(1):25-66, 1967.

18 M. Habib and C. Paul. A survey of the algorithmic aspects of modular decomposition. Computer Science Review, 4(1):41-59, 2010.

19 J. Hopcroft and R. Karp. An n^5/2 algorithm for maximum matchings in bipartite graphs. SIAM Journal on computing, 2(4):225-231, 1973.

20 J. Lanlignel, O. Raynaud, and E. Thierry. Pruning graphs with digital search trees. application to distance hereditary graphs. In STACS, pages 529-541. Springer, 2000.

21 G. Mertzios, A. Nichterlein, and R. Niedermeier. Linear-time algorithm for maximumcardinality matching on cocomparability graphs. arXiv, 2017. arXiv:1703.05598.

22 S. Micali and V. Vazirani. An $O(\sqrt{V} E)$ Algorithm for Finding Maximum Matching in General Graphs. In FOCS'80, pages 17-27. IEEE, 1980. 
23 D. Paulusma, F. Slivovsky, and S. Szeider. Model counting for cnf formulas of bounded modular treewidth. Algorithmica, 76(1):168-194, 2016.

24 M. Rao. Clique-width of graphs defined by one-vertex extensions. Discrete Mathematics, 308(24):6157-6165, 2008.

25 M. Tedder, D. Corneil, M. Habib, and C. Paul. Simpler Linear-Time Modular Decomposition Via Recursive Factorizing Permutations. In ICALP, pages 634-645. Springer, 2008.

26 M.-S. Yu and C.-H. Yang. An $O(n)$-time algorithm for maximum matching on cographs. Information processing letters, 47(2):89-93, 1993. 